\title{
Investigating Differences in Academic Intrinsic, Extrinsic, and Amotivation Among Children of Immigrant Parents
}

Natasha Pooran

College students in the US often experience a pressure to succeed brought on by their unique intrinsic and extrinsic motivations. However, is there a possible connection between these academic motivations and having an immigrant parent? To determine if differences exist in intrinsic, extrinsic, and amotivation among children of immigrant parents (CIPs), I employed a 28-item survey adapted from the Academic Motivation Scale (AMS). I analyzed survey data collected from 183 undergraduate students at the University of Texas at Arlington using three chi-square tests of independence comparing the three types of motivation in CIPs and non-CIPs and a one-way analysis of variance (ANOVA) assessing average scores across types of motivation. Results showed no relationship exists between types of academic motivation and CIP status, but extrinsic motivation scores were significantly higher across all college students. Future studies are needed to examine other factors that may influence academic motivations.

Keywords: academic motivation, intrinsic motivation, extrinsic motivation, amotivation, children of immigrant parents (CIPs).

From my personal experience as a child of Asian immigrants in the US, there is a unique kind of academic motivation brought on by the pressure to take advantage of the academic opportunities my parents never had. Although this is not an openly discussed experience among my peers, many Asian students encounter a "model minority" stereotype that depicts Asians as hardworking, intelligent, and reserved - which can challenge wellbeing and limit adolescents' identity formation (Thompson et al. 108). When attempting to understand why this experience exists for myself, the common denominator among many students, regardless of ethnicity, could be having immigrant parents. There may be an untapped shared immigrant parent experience that affects college students. Parenting styles may differ based on cultural differences in the value of education, which may affect the motivations of many college students and in turn their academic performance.

In this study, I will investigate the differences in motivation in children of immigrant parents (CIPs) to determine if there are empirical data to support the possible effects of a shared experience. Through data collection, I will evaluate the relationships between whether a student is a child of an immigrant parent and their intrinsic motivation, extrinsic motivation, and amotivation. Intrinsic motivation can be defined as a want to know, 
accomplish things, and experience stimulation for personal fulfillment. Extrinsic motivation is motivation to earn an award, praise, or other external reasons. Lastly, amotivation is a complete lack of motivation to complete any task ("The AMS" 1004).

This study will contribute to the body of knowledge concerning the possible relationships between immigrant families and academic motivation. This research is of critical importance now as slightly more than half of all undergraduate college students in the United States are first-generation students, meaning that the students' parents did not have a bachelor's degree (RTI International 1). Many of these first-generation students come from immigrant families across all ethnicities. If this is more understood, then we can develop programs aimed at accounting for the lack of certain motivations in CIPs and non-CIPs.

Previous research has been conducted on academic motivation and cultural identity among CIPs. Urdan and Munoz utilized surveys, implicit association tests, and interviews with 94 college undergraduates who were either CIPs or non-CIPs to determine an association between cultural identity and academic motivation (247). Results showed that CIPs with a strong and positive cultural identity had some motivational benefits and few costs (Urdan and Munoz 247). The study provides a basis for the investigation on whether just being a CIP, regardless of the degree to which an individual identifies with it, may affect academic motivation.

Another study employed survey data and university records of 998 college undergraduates to determine possible relationships between family interdependence and academic adjustment, motivation, and achievement (Tseng 966). Across all ethnic groups, CIPs placed a greater emphasis on family interdependence, which can be defined as emotional, financial, and instrumental support to family members (Tseng 966). Furthermore, Tseng found that this increase in family interdependence was related to increased academic motivation but a decrease in achievement due to greater behavioral demands (966). Such results suggest that having an immigrant family can affect an individual's academic motivation. From these findings and the previous study, this research can determine if academic motivation is a function of immigrant parent status.

Without a doubt, parents have a significant influence on the development of their children. In an interviewbased study on 451 young (ages 6-11) and diverse students, the researchers found that children from immigrant backgrounds develop their centrality (the extent to which they define themselves in terms of ethnicity) and private regard (evaluative beliefs about their ethnicity) based on their parents' racial socialization, or their parents' display of the behaviors, perceptions, values, and beliefs of their ethnic group (Gillen-O'Neel et al. 1470). Additionally, this study found that minority children reported higher levels of intrinsic motivation than their European American peers while accompanied by higher academic anxiety (Gillen-O'Neel et al. 1470). The presence of such findings in a young population may foreshadow that this identity formation and differences in intrinsic motivation may be present at an older age, as it began so early in their academic careers.

Based on the results from the Motivated Strategies for Learning Questionnaire (MSLQ) and a collection of backgrounds and grades of 650 tenth grade students, Blom and Severiens found that there were significant differences between nonimmigrant and immigrant girls' self-regulated deep learning (complex understanding and engagement in critical thinking of knowledge) and surface learning (factual and superficial knowledge) (41). The article states that the differences in the way students learn and their underlying motivational attitudes were influenced by their backgrounds (Blom and Severiens 41). Things such as aspiring for more than their parents' social status, wanting a better job, or support of family values, all factor into the learning of an individual (Blom and Severiens 41). From this, it is reasonable to suggest that college students may exhibit the same differences in academic motivation if one or more of their parents is an immigrant.

In research conducted by Mwangi, Daoud, English, and Griffen, students' academic motivation was seen to be a function of their family's ethnicity and nativity (Mwangi et al. 479). This research also found that parental support could predict the academic goal orientations of students (Mwangi et al. 479). Although this may seem strictly related to extrinsic motivation, the researchers identified that students with immigrant parents had 
internalized their parents' ideas of success in the United States (Mwangi et al. 479). In turn, these students have a higher tendency toward motivation to please their family. As evidenced by this study, the influence of parental guidance is significant as it may change the source of students' intrinsic motivation. Since this finding was seen in Black college students with immigrant parents, it can be hypothesized that intrinsic motivation scores for students with at least one immigrant parent would differ from those without immigrant parents.

To hypothesize regarding the differences in extrinsic motivation in a college-aged population, one must look at the existing research on motivations for millennials. Henstra and McGowan reviewed the personal statements of $\mathbf{4 0}$ graduate students in order to determine millennials' motivation to pursue a career in public services (490). From their review, some of the key motivating factors they found were salary, job security, opportunity for advancement, or flexible work hours (Henstra and McGowan 490). However, they also found equal motivations to pursue a public service career due to an attraction to public policy making, commitment to civic duty and the public interest, compassion, and self-sacrifice (Henstra and McGowan 490). From this study, it seems that these college students were motivated to attend graduate school for equal reasons of passion and practicality. Although the researchers did not collect data on immigrant parent status, this study provides evidence that many students aspire to continue school for both intrinsic and extrinsic reasons. This suggests that we may see similar motivations among the college-aged population tested in this study.

Although students may come from a family or culture which emphasizes the importance of education, this does not always result in an intrinsic motivation, but may in fact create the opposite. In their two experimental studies on Chinese students concerning their intrinsic and extrinsic motivation, Wang et al. state that emphasis on achievement and high standards imposed by Chinese parents resulted in students' relying on extrinsic motivations such as grades to maintain their interest in school (105). It is possible that immigrant parents' overemphasis on the importance of success in education creates extrinsic motivation in their child. Although this is not necessarily a negative consequence, it does show that parental influence can change the way students view their education. Although the researchers do not mention if these students had at least one immigrant parent, the possibilities are high due to their ethnicity. From this, we can hypothesize that having immigrant parents could lead to significant differences in extrinsic motivation as well.

Another age group to consider is middle school-aged children. Unrau and Schlackman investigated the effects of intrinsic and extrinsic motivation on reading achievement for urban middle school students (81). In a multiplegroups structural equation modeling (SEM) analysis of ethnicity, gender, grade level, intrinsic motivation, extrinsic motivation, and reading achievement, Asian and Hispanic students had significant intrinsic and extrinsic motivation scores for reading achievement (Unrau and Schlackman 81). Since the studies of Unrau and Schlackman and Wang et al. regarding middle school students indicate that Asians and Hispanics have both intrinsic and extrinsic motivations, we can hypothesize that CIPs may have significantly different levels of these academic motivations compared to other college students $(81 ; 105)$. Unrau and Schlackman explain that sociocultural processes within schools may contribute to a more accurate explanation of differences in minority student performance (81). Despite a lack of indication of immigrant parent status, the possible connection to sociocultural influences and CIP status allows us to hypothesize that the academic motivations of students with immigrant parents may be significantly different than their non-CIP counterparts.

Based on the literature review and evidence shown for the possibility of CIPs to exhibit significantly different levels of intrinsic and extrinsic motivation, I hypothesize that students with an immigrant parent would show significantly different scores in amotivation as well compared to their counterparts. From the ratings of 51 Chinese fifth-graders about their mothers' parenting and school motivation, Cheung and McBride-Chang came to the conclusion that repeated comparison of their own children's examination results with those of their betterperforming classmates was associated with amotivation and extrinsic motivation (1). In contrast, practices such as parental involvement and vigilance over schoolwork were related to children's intrinsic motivation to learn in 
school (Cheung and McBride-Chang 1). This study provides evidence that parenting affects motivation levels in students. It is not explicitly mentioned if the students had at least one immigrant parent, but the non-American influenced ideals projected onto the Chinese children can be compared to the CIP parenting style. From this, it can be assumed that there will be significant differences in the amotivation of students with immigrant parents.

The participants of the study were a sample of 183 undergraduate students ( 39 males, 144 females) from the University of Texas at Arlington recruited through SONA, the Student Research Participation website. Participants voluntarily signed up to partake in the study after reading a brief description, then proceeded to answer the questions provided. All responses were de-identified to maintain anonymity. Neither gender, race, ethnicity, classification, nor CIP status were factored into participant selection, and those who completed the survey received 0.50 SONA credits as compensation for their efforts.

In an online version of the survey, participants first completed an informed consent page where they were led to believe the study was about the processes underlying why undergraduate students chose to attend college. However, in the debrief it was explained that the true purpose of the study was to evaluate relationships between academic intrinsic, extrinsic, and amotivation and whether a student is a child of an immigrant parent. The true purpose of the study was withheld so that participants' responses to the survey questions were spontaneous and not influenced by having this information.

Following the informed consent page, prompts guided participants to fill out demographic questions including gender (Male, Female), race/ethnicity (White, Black or African American, American Indian or Alaska Native, Asian, Native Hawaiian or Pacific Islander, Other), classification (Freshman, Sophomore, Junior, Senior), and whether one or more of the student's biological parents is an immigrant (Yes, Maybe, No).

Then, the participants completed questions measuring academic intrinsic, extrinsic, and amotivation adapted from the Academic Motivation Scale (AMS) ("AMS-C 28"1). The scale is originally composed of 28-item sevenpoint Likert scales measuring intrinsic, extrinsic, and amotivation; it has high internal and external consistency and is a widely-used measure for motivation (Javaeed et al. 1). In this study, all 28 questions were included in the survey.

Each question had a statement for the participant to answer on a Likert scale from 1 ("Does Not Correspond at All") to 7 ("Corresponds Exactly"). For example, questions 2, 9, 16, and 23 measured the intrinsic motivation "to know." Question 2 was, "Because I experience pleasure and satisfaction while learning new things." Question 9 was, "For the pleasure I experience when I discover new things never seen before." Question 16 was, "For the pleasure that I experience in broadening my knowledge about subjects which appeal to me." Question 23 was, "Because my studies allow me to continue to learn about many things that interest me."

Due to the large required sample size and the extension of online instruction into the Spring 2021 semester, the study took place entirely online. The SONA website directed participants to QuestionPro. After completing the survey questions, a debriefing statement appeared, at which point the participants had the opportunity to opt-out of allowing the use of their data once they learned of the study's true purpose. If participants indicated their consent to the use of their data, responses were recorded anonymously and stored in QuestionPro, following which the participants were dismissed. If participants chose to withdraw their data, the survey was automatically terminated and all responses were deleted. There were no penalties or negative consequences for them if they withdrew from the study. Even if they withdrew from the study, they were still entitled to 0.50 SONA credits. Participation took approximately 5 minutes; all participants received compensation in the form of 0.50 SONA credits, which were automatically delivered upon completion. The data from all participants were then compiled and entered into SPSS for testing. IBM SPSS Release 27.0.0.0 was used to analyze the data collected.

A total of 183 undergraduate students were included in this study and, among them, $144(78.7 \%)$ were females, and 39 (21.3\%) were males. Student ethnicities varied, with 75 (41\%) White, 47 (25.7\%) Asian, 31 (16.9\%) Other, 23 (12.6\%) Black, and 7 (3.8\%) American Indian or Alaskan Native. There were 81 (44.3\%) Freshman in the 
sample, 45 (24.6\%) Sophomores, 35 (19.1\%) Juniors, and 22 (12\%) Seniors. When asked if one or more of their biological parents are immigrants, student responses were relatively equal, with 89 (48.6\%) students answering "Yes", 6 (3.3\%) answering "Maybe", and 88 (48.1\%) answering "No".

To test the hypothesis that differences in intrinsic motivation exists for students with differing immigrant parent status (Yes, $\mu=4.27, \sigma=1.23$, Maybe, $\mu=4.43, \sigma=1.35$, No, $\mu=4.32, \sigma=1.26$ ), a chi-square test of independence was used. A significant difference was not found, $X 2(116, N=183)=94.03, p=0.93, V=0.51$. This does not support the hypothesis that differences in intrinsic motivation exist for students with differing immigrant parent status (see Figure 1).

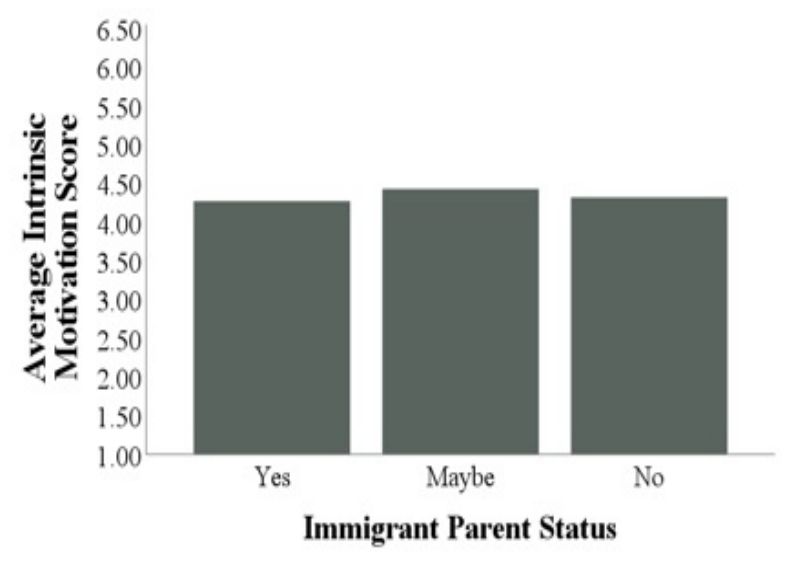

Figure 1.

To test the hypothesis that differences in extrinsic motivation exists for students with differing immigrant parent status (Yes, $\mu=5.49, \sigma=0.87$, Maybe, $\mu=5.10, \sigma=0.76$, No, $\mu=5.14, \sigma=1.10$ ), a chi-square test of independence was used. A significant difference was not found, $X 2(98, N=183)=81.17, p=0.89, V=0.47$. This does not support the hypothesis that differences in extrinsic motivation exist for students with differing immigrant parent status (see Figure 2).

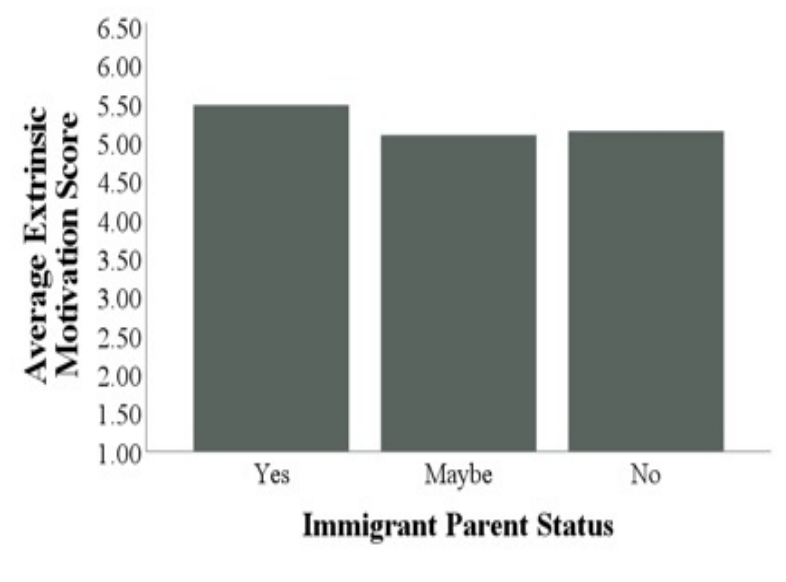

Figure 2.

To test the hypothesis that differences in amotivation exists for students with differing immigrant parent status (Yes, $\mu=2.32, \sigma=1.40$, Maybe, $\mu=2.46, \sigma=1.04$, No, $\mu=2.00, \sigma=1.12$ ), a chi-square test of independence was used. A significant difference was not found, $X 2(42, N=183)=38.33, p=0.63, V=0.32$. This does not 
support the hypothesis that differences in amotivation exist for students with differing immigrant parent status (see Figure 3).

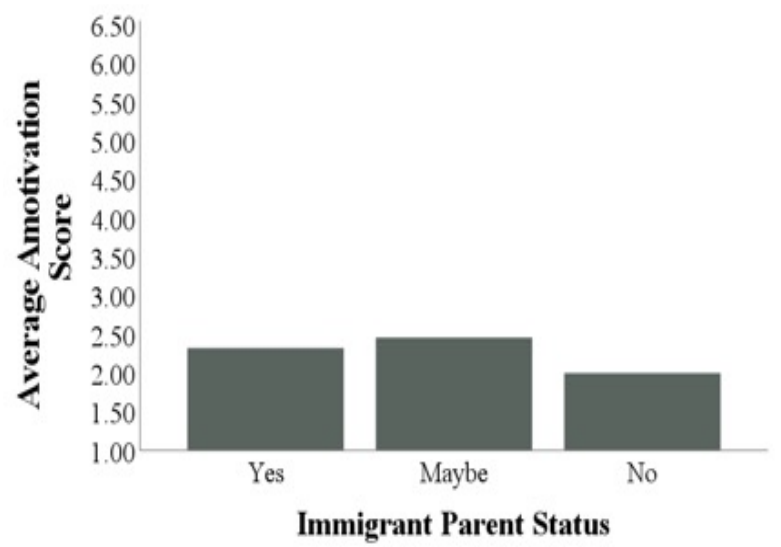

Figure 3.

To assess differences in average academic motivation scores across types of motivation (intrinsic, extrinsic, or amotivation), a one-way randomized analysis of variance (ANOVA) was used. Significant differences were revealed by the ANOVA, $F(2,6)=194.44$, MSE $=0.04, p \approx 0.00, \eta 2=0.99$. This supports the hypothesis that average academic motivation scores differ based on the type of motivation. Bonferroni post-hocs showed that specifically, average academic motivation scores for extrinsic motivation $(M=5.24, S E=0.11)$ were significantly higher than those for intrinsic motivation $(\mathrm{M}=4.34, \mathrm{SE}=0.11)$ and amotivation $(\mathrm{M}=2.26, \mathrm{SE}=0.11)$. Also, average academic motivation scores for intrinsic motivation were significantly higher than those for amotivation. This suggests that for this sample, academic motivation scores were significantly affected by type of motivation (see Figure 4).

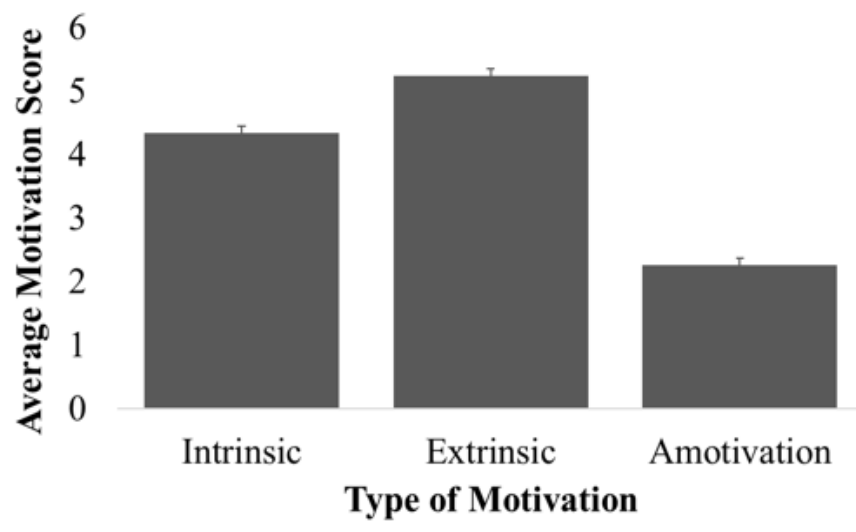

Figure 4.

The goal of this study was to bridge the gap between types of motivations in CIPs to develop a more accurate understanding of how the immigrant parent experience may influence academic success. The current sample of college students and how they compare to relevant literature may help in understanding how differences in types of motivation contribute to students' perspectives on education, and how that affects their performance in school.

The sample we collected supported none of our three hypotheses. However, significance was found for 
motivation scores based on the type of motivation. The significance for the comparison of average motivation scores across types suggests that college students have significantly different levels of intrinsic motivation, extrinsic motivation, and amotivation in regard to academics. More specifically, the sampled college students have significantly higher extrinsic motivation scores in comparison to intrinsic motivation and amotivation scores. This supports previous research that students have similar reasons and motivations for attending college (Henstra and McGowan 490).

In this study, data collection involved self-report surveys from college students that were recruited through UTA's online participant pool, SONA. As all data were collected from college students, the age range and education level were limited for this study. Although beneficial for applying this information to college populations, this limits the ability to apply this research to the general population. The survey aspect also limited the study in that participants may have altered their true answers to socially desirable ones due to the social desirability bias, thus skewing the data and the results obtained. The online format of the survey also potentially limited the accuracy of the data received, as a lack of supervision while completing the survey could have contributed to participants quickly reading through the survey and not giving their true answers. This is evidenced as the estimated amount of time to complete the survey was 30 minutes, but the actual average completion time was 5 minutes, which is not sufficient time to answer 28 Likert scale questions accurately.

For all hypotheses, the current sample appears to be significantly inconsistent with the expectations based on previous research. This implies that extrinsic, intrinsic, and amotivation may not be interconnected with immigrant parent status, and this study can serve as the foundation for further research. In the future, correlational research can be conducted on these variables to see if there is a relationship between one independent variable and academic performance, whether the student is an immigrant, or whether family members other than parents were immigrants. From this, researchers can explore using analysis of variance tests to experiment with multiple variables simultaneously.

To account for the demographic and age limitations of this study, future studies may advertise the survey in off-campus areas with varying demographics such as local dining areas, libraries, and coffee shops. The social desirability bias can be accounted for by assuring the participant that their answers will be kept confidential before they complete the survey, which may increase the likelihood of the participant responding truthfully. Also, offering monetary compensation or converting the online survey into a paper form with researcher supervision during completion can increase the accuracy of survey answers. Attention check questions inserted into the survey could also be a useful tool in determining which participants took a reasonable amount of time to complete the survey accurately. Additionally, the survey data collected in this study regarding participants' gender, ethnicity, and classification can be used for further analysis in possible reasons behind differing academic motivations.

Overall, this study aimed to examine how academic extrinsic, intrinsic, and amotivation in college students could be a function of immigrant parent status. Significance was not found for all types of motivation for students who do have, might have, or do not have immigrant parents, but differences were found in the average scores of different types of motivation across all college students sampled. This research contributes to the body of knowledge concerning types of motivations in college students and provides a basis for further study concerning possible connections to academic motivations such as immigrant parent status, gender, ethnicity, classification, and more. From this, researchers can determine what other internal or external factors contribute to students' academic motivations. 


\section{References}

Blom, S., \& Severiens, S. "Engagement in self-regulated deep learning of successful immigrant and non-immigrant students in inner city schools." European Journal of Psychology of Education, 23(1), 2018, pp. 41-58, http://www.jstor.org/stable/23421617.

Cheung, C., \& McBride-Chang, C. (2008). "Relations of perceived maternal parenting style, practices, and learning motivation to academic competence in Chinese children." Merrill-Palmer Quarterly, 54(1), 2008, pp. 1-22, http://www.jstor.org/stable/23096077.

Gillen-O'Neel, C., Ruble, D., \& Fuligni, A. "Ethnic stigma, academic anxiety, and intrinsic motivation in middle childhood." Child Development, 82(5), 2011, pp. 14701485, http://www.jstor.org/stable/41289858.

Henstra, D., \& McGowan, R. "Millennials and public service: An exploratory analysis of graduate student career motivations and expectations." Public Administration Quarterly, 40(3), 2016, pp. 490-516, http://www.jstor.org/stable/24772880.

Javaeed, A., Asghar, A., Allawat, Z., Haider, Q., Mustafa, K. J., \& Ghauri, S. K. "Assessment of Academic Motivation Level of Undergraduate Medical Students of Azad Kashmir, Pakistan." Cureus, 11(3), 2019, p. e4296, doi:10.7759/cureus.4296.

Mwangi, C. A. G., Daoud, N., English, S., \& Griffen, K. A. "'Me and My Family': "Ethnic differences and familial influences on academic motivations of Black collegians." The Journal of Negro Education, 86(4), 2017, pp. 479-493, http://www.jstor.org/stable/10.7709/ jnegroeducation.86.4.0479.

RTI International. First-generation college students: Demographic characteristics and postsecondary enrollment. Washington, DC: NASPA, 2019, https://firstgen. naspa.org/files/dmfile/FactSheet-01.pdf.

Thompson, T. L., Kiang, L., \& Witkow, M. R.. "'You're Asian; You're supposed to be smart': Adolescents' experiences with the Model Minority Stereotype and longitudinal links with identity." Asian American Journal of Psychology, 7(2), 2016, pp. 108-119, https://doi. org/10.1037/aap0000038.
Tseng, V. "Family interdependence and academic adjustment in college youth from immigrant and U.S.-born families." Child Development, 75(3), 2004, pp. 966-983. doi:10.1111/j.1467-8624.2004.00717.x.

Unrau, N., \& Schlackman, J. "Motivation and its relationship with reading achievement in an urban middle school." The Journal of Educational Research, 100(2), 2006, pp. 81-101, http://www.jstor.org/stable/27548165.

Urdan, T., \& Munoz, C. "Multiple contexts, multiple methods: A study of academic and cultural identity among children of immigrant parents." European Journal of Psychology of Education, 27(2), 2012, pp. 247-265, doi:10.1007/s10212-011-0085-2.

Vallerand, R.J., Pelletier, L. G., Blais, M.R., Briere, N. M., Senecal, C. B., \& Vallieres, E. F. "Academic motivation scale (AMS-C 28) college version." Educational and Psychological Measurement, 52. 1992, https:// www.researchgate.net/profile/Stephen_Joy/post/Does_ anyone_know_where_I_can_get_the_Academic Motivation_Scale-College_version_AMS-C_for_my research/attachment/59d61fa479197b807797e16b/AS \%3A285951063543808\%401445187454068/download/ Academic+Motivation+Scale+College+Version.pdf

Vallerand, R.J., Pelletier, L. G., Blais, M.R., Briere, N. M., Senecal, C. B., \& Vallieres, E. F. "The academic motivation scale: A measure of intrinsic, extrinsic, and amotivation in education." Educational and Psychological Measurement, vol. 52 (4), 1992, pp. 1003-1017, doi:10 $.1177 / 0013164492052004025$.

Wang, Z., Hu, X., \& Guo, Y. "Goal contents and goal contexts: Experiments with Chinese students." The Journal of Experimental Education, 81(1), 2013, pp. 105-122, doi:10.2307/26594370. 